MOTOR NEURON DISEASE

\section{Reactive microglia protect neurons in ALS}

Reactive microglia are crucial for the survival of motor neurons in amyo-

To my surprise, trophic lateral sclerosis (ALS), a new that's when

we saw the

dramatic

changes in

microglia that

we had been

looking for

study has shown. The findings con-

tradict the prevailing view in the field and could inform the development of novel therapies for ALS.

Reactive microglia are thought to have neurotoxic effects in a variety of neurodegenerative diseases, but recent research has indicated that their role is more complex than was previously believed. A number of anti-inflammatory therapies have been developed to target harmful effects of microglia in ALS, but, thus far, none of these agents have been successfully translated to the clinic.

This disappointment could stem from the fact that much of the pre-

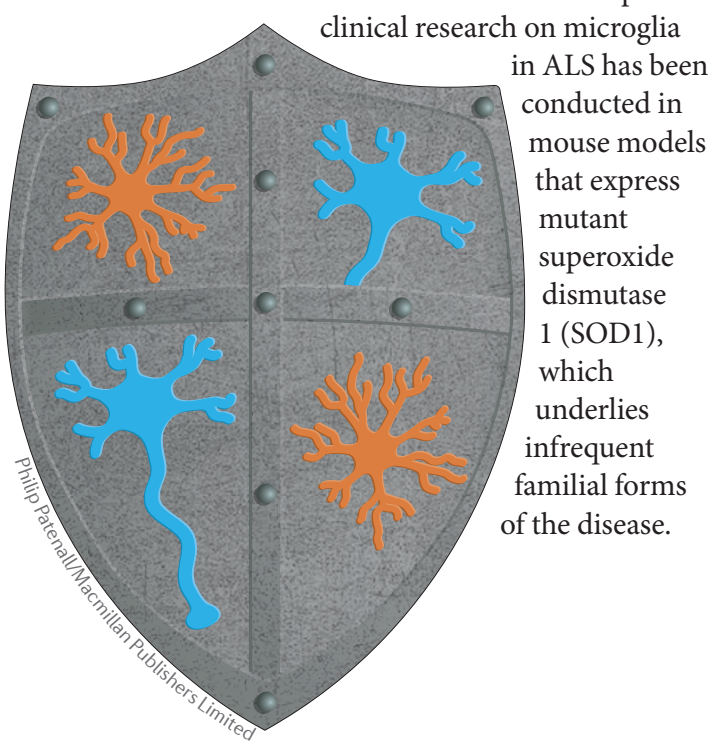

In the new study, the investigators examined a mouse model that recapitulates the TAR DNA-binding protein 43 (TDP43) pathology seen in the vast majority of patients with ALS. Neurons in these mice express an inducible transgene that encodes a mutant form of human TDP43 with a broken nuclear localization signal that causes the protein to localize to the cytoplasm and form aggregates. This aggregation leads to motor neuron degeneration, but switching off the transgene reverses this damage.

Unexpectedly, the researchers did not observe any changes in the number or morphology of microglia while human TDP43 was being expressed in the animals, but after the transgene was switched off, the microglia dramatically proliferated and changed to a reactive morphology. "To my surprise, that's when we saw the dramatic changes in microglia that we had been looking for," remarks lead author Krista Spiller.

When the team examined spinal cord tissue from individuals with ALS, they observed an increase in numbers of reactive microglia in patients with a SOD1 mutation but not in those with sporadic or C9orf72-linked ALS. This finding suggests that the mouse model replicates the features of non-SOD1-associated ALS.

The researchers examined the transcriptome of the microglia from their transgenic mice after TDP43 expression was switched off, and they found that the cells upregulated hundreds of genes, including many associated with phagocytosis. Interestingly, the team also observed that the reactive microglia clustered around neurons and contained human TDP43 but not other neuronspecific proteins, suggesting that the microglia help to clear TDP43 aggregates in these animals.

Importantly, treatment with PLX3397, an agent that eliminates microglia, prevented recovery of the mice after the transgene was turned off and also blocked the clearance of TDP43 in the animals. "The proliferation of reactive microglia was critical for the survival of motor neurons, rather than contributing to their death," explains Spiller.

Spiller and colleagues now want to find out why the reactive microglia clear TDP43 aggregates after expression of the transgene is halted but not while expression is ongoing. "The hope is that if we could encourage this type of pro-clearance microglial response during disease, then the progression could be slowed down or even stopped," Spiller concludes.

Charlotte Ridler

ORIGINAL ARTICLE Spiller, K. J. et al. Microgliamediated recovery from ALS-relevant motor neuron degeneration in a mouse model of TDP-43 proteinopathy. Nat. Neurosci. https://doi. org/10.1038/s41593-018-0083-7 (2018) 